Revisão/Review

\title{
Caracterização da ativação plaquetária nos concentrados de plaquetas por citometria de fluxo ${ }^{7}$ \\ Flow cytometric characterization of platelet activation in platelet concentrates
}

Evaldo P. Landi ${ }^{1}$

José F. C. Marques Júnior ${ }^{2}$

\begin{abstract}
A caracterização da expressão das glicoproteínas de superfície plaquetária esuperfície pró-coagulante por citometria de fluxo éuma técnica sensível para identificação da ativação plaquetária em concentrados de plaquetas. A ativação plaquetária pode desen cadear alterações que irão influir na viabilidade das plaquetas du rante o período de estocagem. A detecção da ativação plaquetária durante o preparo e estocagem dos concen tra dos de plaquetas pode permitir a identificação dos mecanismos de ativação e sugerir alterações que irão influir na qualidade final do produto. A utilização isolada de anticorpos monoclonais específicos para plaquetas ativadas pode levar a erros de interpretação. Assim, é aconselhável o uso de painéis de anticorpos ca pazes de avaliar a migração das glicoproteín nas IIb-IIla e Ib-IX, o processo de degranulação e a formação de superfície prócoagulante. Rev. bras. hematol. hemoter. 2003;25(1):39-46.
\end{abstract}

Palavras-chave: Concentrado de plaquetas; ativação plaquetária; citometria de fluxo.

\section{Introdução}

A transfusão de concentrados de plaquetas é fundamental para o controle e prevenção de hemorragias nos pacientes trombocitopênicos. A resposta terapêutica obtida com a transfusão irá depender, entre outros fatores, da integridade da função biológica das plaquetas após a coleta, do processamento e da estocagem. A detecção da ativação plaquetária em concentrados de plaquetas pode indicar que pelo menos parte da função biológica está comprometida.

Com o intuito de avaliar a viabilidade, a ativação, a presença de lise e a função plaquetária foram propostos vários testes in vitro., ${ }^{1,2}$ Os testes que avaliam a viabilidade das plaquetas são os que apresentam a melhor correlação com a sobrevida in vivo após a transfusão. ${ }^{2-5}$ Eles se baseiam na avaliação da morfologia esférica ou discóide das plaquetas por microscopia óptica ou espectofotometria, ${ }^{6}$ na resposta ao estresse hipotônico ${ }^{7}$ e na capacidade de mudança da forma discóide para esférica após estimulação por agonista. ${ }^{8}$

A detecção da ativação plaquetária pode avaliar precocemente as alterações que irão influir na viabilidade das plaquetas durante 0 período de estocagem. ${ }^{9,10} \mathrm{~A}$ ativação plaquetária pode ser ava-

${ }^{1}$ Pós-graduando da Disciplina de Hematologia do Departamento de Clínica Médica da Unicamp.

${ }^{2}$ Médico Hematologista/Hemoterapeuta da Divisão de Hemoterapia do Centro de Hematologia e Hemoterapia da Unicamp.

Correspondência para: Evaldo Pasquini Landi

Cidade Universitária Zeferino Vaz

Rua Dr. Carlos Chagas, 480 - Caixa Postal 6198 - CEP: 13083-970 - Campinas-SP - Brasil

Tel: (019) 3788-8605 - e-mail: langa20@hotmail.com 
liada pela dosagem plasmática de proteínas plaquetárias específicas, como a $\beta$-tromboglobulina, fator plaquetário 4 e metabólitos do tromboxane A2 e pela expressão das glicoproteínas de superfície e superfície pró-coagulante por citometria de fluxo. ${ }^{11,12}$ Além disso, o estudo por citometria de fluxo permite avaliar a expressão das glicoproteínas de superfície e da superfície pró-coagulante antes e após a adição de agonista, determinando o potencial de resposta das plaquetas. ${ }^{13}$

As aplicações clínicas, as metodologias de preparo e as principais falhas na caracterização da função plaquetária por citometria de fluxo já foram revistas, ${ }^{14,15}$ porém, a melhor combinacão de anticorpos monoclonais e marcadores da ativação plaquetária para o estudo dos concentrados de plaquetas ainda não foi definido.

Este artigo revê as características, a função biológica e a expressão das principais glicoproteínas e fosfolípides envolvidos no processo de ativação plaquetária nos concentrados de plaquetas e procura estabelecer um painel de anticorpos monoclonais para o estudo da ativação plaquetária por citometria de fluxo.

Principais marcadores da ativação plaquetária:

\section{Glicoproteínas de membrana}

A. Glicoproteína (GP) IIb-IIIa (CD41/CD61)

A GP Ilb-IIla pertence à família das integrinas e é a glicoproteína mais abundante na superfície plaquetária, com cerca de 50.000 cópias por plaqueta. ${ }^{16}$ Como outras moléculas da família das integrinas, a GP IIb-IIla atua como um receptor na interação entre plaquetas ou na ligacão das plaquetas com proteínas do subendotélio vascular, decisivos para o processo de agregação e adesão, respectivamente. ${ }^{16} \mathrm{~A}$ tabela 1 sumariza as principais integrinas presentes na membrana plaquetária e suas funções.

A GP IIb-Illa é constituída por um complexo de cadeias de polipeptídeos. A GP IIb é composta pelas subunidades $I \mathrm{lb} \alpha$ e $\mathrm{Ilb} \beta$ ligadas por pontes dissulfeto, com peso molecular de $116 K D$ e $25 K D$, respectivamente. Apenas a subunidade $\operatorname{Ilb} \beta$ apresenta porção citoplasmática. ${ }^{17} 0$ polipeptídeo IIIa ( $\beta 3$ ) apresenta peso molecular de $100 \mathrm{KD}$, porção cito plasmática e está associado à GP IIb por ligação não covalente. ${ }^{17}$ Além de estar presente na membrana plaquetária, a GP IIb-IIla também é encontrada no sistema canalicular aberto (SCA) e na membrana dos grânulos alfa. 18,19 Estima-se que 0 equivalente a $100 \%$ da GP IIb-IIla presente na superfície plaquetária possa ser mobilizado do SCA e grânulos alfa durante o processo de ativação. ${ }^{20}$

A ativação plaquetária desencadeia mudanças conformacionais na GP IIb-IIIa, que passa a funcionar como um receptor capaz de se ligar à seqüência de aminoácidos arg - gly - asp (RGD) presente nas moléculas de fibrinogênio, fator de von Willebrand (FVW), fibronectina e vitronectina, e à seqüência Lys - GIn - Ala - Gly - Asp - Val (LGAGAV) do fibrinogênio. ${ }^{21,22} 0$ sítio principal de ligação da seqüência RGD é a porção amino terminal da subunidade $\beta 3$ (GPIIIa).22

Os anticorpos monoclonais que identificam a GP IIb-IIla podem reconhecer epítopos na forma não ativada das diferentes subunidades

Tabela 1

Glicoproteínas da família das integrinas presentes nas plaquetas

\begin{tabular}{cccc}
\hline Designação & Subunidades & Cluster de diferenciação & Função biológica \\
\hline GP la-lla ou VLA-2 & $\alpha 2 \beta 1$ & CD49b/CD29 & Receptor do colágeno \\
GP IC-lia ou VLA-5 & $\alpha 5 \beta 1$ & CD49e/CD29 & Receptor da fibronectina \\
VLA-6 & $\alpha 6 \beta 1$ & CD49f/CD29 & Receptor da laminina \\
GP Illb-llla & $\alpha$ Rllb $\beta 3$ & CD41/CD61 & $\begin{array}{c}\text { Receptor do fibrinogênio, } \\
\text { fabronectina, vitronectina } e\end{array}$ \\
& $\alpha$ fator de von Willebrand (FVW) & Receptor da vitronectina \\
\hline
\end{tabular}


do complexo. ${ }^{23}$ Há também anticorpos mono clonais que reconhecem a conformação da GP IIb-IIla ativada ou ligada à molécula de adesão. ${ }^{24}$ Os anticorpos do tipo PAC1 reconhecem especificamente a conformação ativada da GP IIb IIIa, os anticorpos do tipo LIBS (Ligand-Induced Binding Sites) reconhecem epítopos da GPIIbIlla quando ligada à seqüência RGD ou à molécula de fibrinogênio, e os anticorpos do tipo RIBS (Receptor-Induced Binding Sites) reconhecem a molécula de fibrinogênio ligada à GPIIb-IIIa. ${ }^{24-26}$

A utilização de anticorpo monoclonal que identifique a GP IIb-IIla na forma não ativada permite avaliar a mobilização do pool interno pelo aumento da expressão da glicoproteína após a ativação plaquetária, além de identificar micropartículas derivadas da membrana plaquetária. ${ }^{27-30}$ Nos estudos in vitro, define a população de plaquetas na presença de outro anticorpo monoclonal e avalia a expressão da glicoproteína após a estimulação das plaquetas. ${ }^{13}$

Os anticorpos monoclonais que reconhecem a configuração ativada da GPIIb-IIIa podem fornecer informações adicionais quando analisados em conjunto com anticorpos para a configuração não ativada da glicoproteína.

A reatividade pode ser expressa em porcentagem de plaquetas positivas e/ou intensidade média de fluorescência. ${ }^{14}$

\section{B. Complexo GPIb-IX-V}

A glicoproteína Ib (GPIb) pertence à família das glicoproteínas ricas em leucina ( $G R L)$, atua como receptor para o FVW do subendotélio vascular em condições de fluxo (shear stress) e como receptor de trombina. ${ }^{31,32}$ É composta pelas subnidades GPIb $\alpha$ de 150KDa e GPIb $\beta$ de 27KDa ligadas por pontes dissulfeto e forma um complexo por ligação não covalente com as glicoproteínas IX e V na proporção de 1:1 (GPIX: GPIb) e 1:2 (GPV: GPIb).,32,33 Está presente na membrana plaquetária, no SCA, nos grânulos alfa e nos grânulos densos. ${ }^{16}$ Estima-se cerca de 25.000 cópias do complexo por plaqueta. ${ }^{34}$

0 FVW liga-se à porção N-terminal da subunidade alfa, aminoácidos His1 - Gly282, enquanto a trombina se liga e, em seguida, cliva a GPV. ${ }^{32,}{ }^{34}$ A ativação da GPIb estimula as mo- léculas citoplasmáticas 14-3-3, fosfatidilinositol 3-kinase e calmodulina, que irão aumentar a concentração intracitoplasmática de cálcio e promover a ativação da GPIIb-IIla, que irá potencializar o processo. 0 resultado da estimulação será a mudança da forma discóide para a esférica, a liberação dos grânulos alfa e lisossomais, um maior potencial de agregação e o início da contração plaquetária. 35,36

Os anticorpos monoclonais que identificam o complexo GPIb-IX-V podem reconhecer in dependentemente cada uma das subunidades do complexo, como a GPIX (CD42a), a GPIb $\alpha$ (CD 42b), a GPIb $\beta$ (CD 42c) e a GPV (CD 42d). ${ }^{23}$ A maioria dos estudos utiliza anticorpos que reconhecem a GPIb. ${ }^{37,38}$

As plaquetas em suspensão podem apresentar $60 \%$ a $80 \%$ de redução da expressão do complexo GPIb-IX-V. ${ }^{39} 0$ fenômeno pode ser reversível, não afeta a capacidade de adesão plaquetária em condições de fluxo e pode ser atribuído à redistribuição do complexo no SCA (internalização) e/ou à lise da GPV pela trombina. ${ }^{39}$

A análise da GPIb em conjunto com a GPIIb-IIla pode fornecer informações mais precisas sobre a migração das glicoproteínas associada à ativação plaquetária. Por tratar-se de glicoproteína específica das plaquetas e que não altera a conformação após a ativação, pode também ser utilizada na identificação de micropartículas derivadas da membrana plaquetária e na definição da população de plaquetas na presença de outro anticorpo monoclonal ou em estudos de estimulação in vitro. Porém, a migração do complexo para o SCA pode levar à diminuição da expressão e falsear os resultados do estudo.

0 complexo é geralmente avaliado pela intensidade média de fluorescência e não pela porcentagem de células positivas porque a redução da expressão é geralmente insuficiente para ocasionar a ausência completa de fluorescência. ${ }^{14}$

\section{Selectinas}

A família das selectinas é composta pelas proteínas ELAM-1 (E-selectina; CD62E) presente nas células endoteliais, LAM-1 (L-selectina; CD62L) presente nos linfócitos e GMP-140 (Pselectina; CD62P) presente nos grânulos alfa 
das plaquetas e nos corpúsculos de WeibelPalade das células endoteliais. ${ }^{16}$ Atuam como receptores de superfície capazes de mediar a interação entre plaquetas ativadas e células endoteliais com neutrófilos e monócitos. ${ }^{40}$

A GMP-140 se liga a carboidratos da membrana, como a fucose e o ácido siálico (sialil-3fucosil-N-acetil lactosamina - Slex; CD 15S).40,41 Apresenta grupamento carboxila intracitoplasmático e é composta por nove repetições de proteínas homólogas às proteínas reguladoras do sistema complemento, uma porção similar ao fator de crescimento epidérmico e uma porção terminal com cerca de cem aminoácidos contendo 0 sítio de adesão. ${ }^{42,43} \mathrm{~N}$ as plaquetas não ativadas, a GMP-140 está localizada na membrana dos grânulos alfa e, após a ativação, passa a ser expressa na membrana plaquetária. Estima-se que haja cerca de 10 mil sítios de ligação da GMP-140 na superfície das plaquetas ativadas. ${ }^{44}$

Vários anticorpos monoclonais específicos para a GMP-140 foram desenvolvidos. ${ }^{42-45} \mathrm{~A}$ glicoproteína é considerada um excelente marcador da ativação plaquetária, e a expressão se correlaciona muito bem com o tempo de estocagem dos concentrados de plaquetas, a expressão da GPIIb-IIIa e a liberação de beta-tromboglobulina. ${ }^{11}$ Porém, as plaquetas degranuladas perdem rapidamente a expressão da GMP-140 na superfície, apesar de permanecerem na circulação e manterem a capacidade de se aderir ao subendotélio. ${ }^{46}$ Portanto, o uso isolado do anticorpo anti-CD 62p pode ocasionar erros de interpretação nos estudos de ativação, a menos que se dose a GMP-140 solúvel no plasma concomitantemente. ${ }^{47}$

A GMP-140 é avaliada pela porcentagem de plaquetas positivas, no entanto a combinação da porcentagem de plaquetas positivas com a intensidade média da fluorescência pode fornecer resultados mais precisos. ${ }^{30}$

\section{Quadraspaninas}

Também chamadas de tetraspaninas, são proteínas que atravessam a membrana celular quatro vezes e apresentam o grupamento carboxila e amina na porção intracitoplasmática. ${ }^{16} \mathrm{~N}$ as plaquetas, são representadas pela proteína p24/CD 9 com 228 aminoácidos e pela granulofisina (CD63; LAMP-3) de 53 KD. ${ }^{23,48}$

A expressão da proteína $p 24 / C D 9$ independe do grau de ativação plaquetária, está associada à GPIIb-IIIa e à GPIV (CD 36) e a sua função biológica está relacionada à ativação plaquetária na presença de íons cálcio. ${ }^{49}$ Apesar de não ser um marcador da ativação plaquetária, a expressão da proteína p24/CD 9 em função da expressão da GPIIb-IIIa, GPIb e GMP-140 ainda não foi avaliada.

A granulofisina (CD 63, LAMP-3) está presente na membrana dos grânulos lisossomais e nos grânulos densos das plaquetas não ativadas. ${ }^{50}$ Cerca de 10 mil sítios de ligação podem ser expressos na superfície da membrana plaquetária após a ativação. ${ }^{48}$ É considerada um excelente indicador da ativação plaquetária, com resultados muito parecidos aos encontrados com a GMP-140, porém com menor intensidade de reação. ${ }^{37,51}$

A presença da granulofisina é avaliada pela porcentagem de plaquetas positivas. ${ }^{30}$

\section{E. Outras}

1- Glicoproteína IV

A glicoproteína IV (CD 36; GPIIIb) apresenta peso molecular de 88KD e não tem homologia com qualquer outra proteína, portanto não é enquadrada em nenhuma família de glicoproteína. Pode ser encontrada em monócitos, células endoteliais, células de melanoma, eritroblastos e plaquetas. ${ }^{23}$ Há cerca de 27 mil moléculas de GPIV por plaqueta, sendo que metade delas se encontra na superfície. ${ }^{52} \mathrm{~A}$ sua função biológica não é inteiramente conhecida, mas pode atuar como receptor para trombospoidina e colágeno ou como molécula de adesão ao subendotélio vascular. ${ }^{53}$

Os anticorpos monoclonais específicos para a GPIV (anti-CD36) ligam-se às plaquetas não ativadas, porém a porcentagem de positividade pode aumentar após a ativação. ${ }^{37,51}$

2- Proteínas lisossomais de membrana, LAMP-1 E LAMP-2

A LAMP-1 e a LAMP-2 (CD 107a e CD 107b) são proteínas homólogas com cerca de $110 \mathrm{KD}$. A expressão depende da ativação plaquetária, 
porém, com cerca de mil moléculas por plaqueta, ela é considerada fraca. ${ }^{53}$ Podem ser utilizados como marcadores da ativação plaquetária em concentrados de plaquetas, mas são substituídos com vantagens pela granulofisina e GMP-140.

II. Desenvolvimento de superfíce prócoagulante

A. Ligação do fator $V$ ativado (FVa)

O FVa liga-se à fosfatidilserina da superfície lipídica das plaquetas ativadas por meio de ligações de alta afinidade. Na presença de íons cálcio, ocorre a ligação do fator $X$ ativado ( $F X a$ ) ao FVa e a conseqüente conversão da protrombina em trombina. O FVa é expresso em níveis muito baixos na superfície das plaquetas não ativadas, porém pode estar presente em níveis significativos nas plaquetas em suspensão que sofreram ativação. ${ }^{54,55}$

0 protótipo do anticorpo é conhecido como V237 e tem sido utilizado com sucesso na avaliação da ativação plaquetária in vivo e in vitro. A porcentagem de plaquetas positivas apresenta boa correlação com outros marcadores da ativação. . $^{13,30,51}$

\section{B. Anexina V}

Assim como outras membranas celulares, a membrana plaquetária apresenta distribuição assimétrica dos fosfolípides de superfície. Em plaquetas não ativadas, $90 \%$ do total da fosfatidilserina presente na membrana está localizado na porção interna da camada bilipídica. ${ }^{56} \mathrm{Na}$ ativação plaquetária, a maior parte da fosfatidilserina passa para a camada externa da membrana e forma uma superfíce prócoagulante capaz de potencializar a formação de trombina. ${ }^{57}$

$A$ anexina $V$ é uma proteína de 37KD com capacidade de se ligar fortemente aos fosfolípides de carga negativa na superfície celular na presença de íons cálcio. Nos estudos de ativação plaquetária, a anexina $\mathrm{V}$ se liga à superfície das plaquetas ativadas e é considerada um marcador sensível, à medida que a porcentagem de plaquetas positivas para anexina $\mathrm{V}$ apresenta relação direta com a expressão das glicoproteínas CD62p e CD63. ${ }^{58,59}$

\section{Discussão}

A ativação plaquetária nos concentrados de plaquetas pode ser caracterizada por citometria de fluxo, utilizando-se anticorpos monoclonais específicos. A avaliação citométrica pode considerar a medida direta da porcentagem de expressão e/ou a intensidade média de fluorescência de um determinado anticorpo, ou a reatividade das plaquetas após a estimulação in vitro. ${ }^{60}$ Podem ser analisados a expressão quantitativa de um antígeno, a presença de alterações conformacionais de uma glicoproteína, os produtos da secreção e da degranulação das plaquetas, a perda do assimetrismo dos fosfolípides de membrana, a ligação com fatores da coagulação e a formação de micropartículas.

Há evidências de que os principais receptores da membrana plaquetária, a GPIb e a GPIIb-IIla, movem-se em direções opostas durante o processo de ativação, acarretando diminuição e aumento da expressão, respectivamente. Do ponto de vista fisiológico, a movimentação pode significar a diminuição da capacidade adesiva (GPIb) e maior potencial para agregação (GPIIb-IIIa). ${ }^{61}$

0 uso da citometria de fluxo e anticorpos monoclonais específicos para detecção da ativação plaquetária é uma técnica sensível, de fácil preparo e boa reprodutibilidade. Utiliza pequenas amostras e exige pouca manipulação, o que evita a ativação associada ao manuseio da amostra. Pode substituir a técnica de dosagem do fator plaquetário 4 , beta-tromboglobulina e metabólitos do tromboxane $A 2$.

Alguns cuidados, porém, devem ser tomados quanto ao preparo das amostras e a interpretação dos resultados. A expressão das glicoproteínas da membrana plaquetária pode variar conforme o tipo de anticoagulante empregado, a solução tampão utilizada para o preparo, o tempo gasto no processamento da amostra até a fixação com paraformaldeído, a presença de fibrina ou FVW na solução e a natureza do anticorpo monoclonal empregado. Além disso, deve ser considerado o investimento inicial na aquisição de um citômetro de fluxo, a necessidade de calibrações freqüentes e de pessoal técnico especializado. 
Na caracterização da ativação plaquetária por citometria de fluxo, podem-se obter resultados mais fidedignos estudando-se paralelamente mais de uma glicoproteína de superfície, uma proteína granular e a formação da superfície pró-coagulante. Se for considerada a elaboração de um painel de anticorpos monoclonais, a caracterização da GPIIb-Illa é particularmente importante, à medida que favorece a identificação das plaquetas e a presença de micropartículas derivadas da membrana plaquetária. Podem ser utilizados anticorpos monoclonais tipo PAC1, RIBS ou LIBS para identificação da conformação da GPIIb-IIla ativada.

Os anticorpos que reconhecem epítopos da GPIIb-IIla não ativada podem ser úteis na caracterização da externalização da glicoproteína associada à ativação. A caracterização da GPIb irá fornecer informações adicionais quanto à identificação das plaquetas e micropartículas de plaquetas em uma solução e o tráfego de glicoproteínas associado à ativação plaquetária.

Para a caracterização da degranulação ou secreção pode ser avaliada a expressão da GMP140 (anti-CD 62p) ou da granulofisina (anti-CD 63). A formação da superfície pró-coagulante é caracterizada pela ligação da anexina $\mathrm{V}$ e/ou FVa à superfície plaquetária. A técnica permite identificar como ativadas as plaquetas que sofreram degranulação, perderam a expressão da GMP140 e granulofisina na superfície e mantiveram a capacidade de se manter na circulação e aderir, sendo que, de outra forma, essas plaquetas seriam interpretadas como se não tivessem sofrido qualquer ativação.

\section{Abstract}

Flow cytometric characterization of platelet membrane glycoprotein expression and procoagulant surface isa sensitive technique to identify platelet activation in platelet concentrates. Platelet activation could contribute to platelet storage lesion reducing platelet viability and its hemostatic function. Detection of activation in platelet concentrates may help to determine the mechanism by which platelets are activated during preparation and storage. It may also suggest methods of intervention to prevent and reduce the platelet storage lesion. A monoclonal antibody panel that evaluates glycoproteins IIb-IIIa and Ib-IX migration, platelet degranulation and platelet procoagulant surfacewould provide more information about platelet activation than activation-dependent monoclonal antibodies alone. Rev. bras. hematol. hemoter. 2003;25(1):39-46.

Key words: Platelet concentrates; platelet activation flow cytometry.

\section{Referências Bibliográficas}

1. Wiliamson LM. Consensus conference on platelet transfusion: How should the safety and efficacy of platelet transfusion be assured? Blood Reviews 1998; 12:203-14.

2. Murphy S, Rebulla P, Bertolini F. In vitro assessment of the quality of stored platelet concentrates. Trans Med Rev 1994;VIII:29-38.

3. Bertolini F, Murphy S. A multicenter evaluation of reproducibility of swirling in platelet concentrates. Transfusion 1994:34:796-801.

4. Bertolini F, Murphy S. A multicenter inspection of the swirling phenomenon in platelet concentrates prepared in routine practice. Transfusion 1996;36:128-132.

5. Holme S, Moroff G, Murphy S. A multi-laboratory evaluation of in vitro platelet assays: the tests for exten of shape change and response to hypotonic shock. Transfusion 1998;38:31-40.

6. Currie LM, Livesey SA, Harper JR, Connor J Cryopreservation of single-donor platelets with a reduced dimethyl sulfoxide concentration by the addition of second-messenger effectors: enhanced retetion of on vitro functional activity. Transfusion 1998:38:160-7.

7. Valeri $C R$, Feingold $H$, Marchionni LD. The relation between response to hypotonic stress and the ${ }^{51} \mathrm{C}$ recovery in vivo of preserved platelets. Transfusion 1974;14:331-7.

8. Holme S, Murphy S. Quantitative measurements of platelet shape by light transmission studies; application to storage of platelets for transfusion. I Lab Clin Med 1978;92:53-64.

9. Metcalfe P, Williamso LM, Reutelingsperger CPM, Swann I, Ouwehand WH, Goodall AH. Activation during preparation of therapeutic platelets affects deterioration during storage: a comparative flow cytometric study of during storage: a comparative flow cytometric study of
different production methods. Br J Haem 1997;98:86-95.

10. Holme S, Sweeney JD, Sawyer S, Elfath MD. The expression of $p$-selectin during collection, processing, and storage of platelet concentrates: relationship to loss of in vivo viability. Transfusion 1997;37:12-17.

11. Rinder HM, Snyder EL, Bonan JL, Napychank PA, Malkus $H$, Smith BR. Activation in stored platelet concentrates: correlation between membrane expression of $p$-selectin 
glycoprotein IIb/IIla, and $\beta$-thromboglobulin release. Transfusion 1993;33:25-9.

12. Rinder HM, Ault KA. Platelet activation and its detection during the preparation of platelets for transfusion. Trans Med Rev 1998;12:271-87.

13. Barnard MR, Macgregor H, Ragno G, Pivacek LE, Khuri $S F$, Michelson $A D$, Valeri CR. Fresh, liquid-preserved, and cryopreserved platelets: adhesive surface receptors and membrane procoagulant activity. Transfusion 1999:39:880-88.

14. Schmitz G, Rothe G, Ruf A, Barlage S, Tschöpe D Clemetson KJ, et al. European working group on clinica cell analysis: consensus protocol for the flow cytometric characterisation of platelet function. Thromb Haemost 1998;79:885-96

15. Michelson AD. - Flow cytometry: a clinical test of platelet function. Blood 1996;87:4925-36.

16. Charo IF, Kieffer N, Phillips DR. Platelet membrane glycoproteins. In: Colman W, Hirsh J, Marder VJ, Salzman EW. (ed.) - Hemostasis and Thrombosis: Basic Principles and Clinical Practice. $3^{\text {rd }}$ ed. Philadelphia, Lippincott Company, 1994. p. 489-507.

17. Lefkovits J, Plow EF, Topol EJ. Platelet glycoprotein Ilb/IIla receptors in cardiovascular medicine. $N$ Engl J Med 1995:332:1553-59.

18. Cramer EM, Savidge GF, Vainchenker W, Berndt MC, Pidard D, Caen JP, Masse JM, Breton-Gorius J. Alphagranule pool of glycoprotein IIb-IIla in normal and pathologic platelets and megakaryocytes. Blood 1990; 75:1.220-7

19. Wencel-Drake JD, Plow EF, Kunicki TJ, Woods VL, Keller $\mathrm{DM}$, Ginsberg MH. Localization of internal pools of membrane glycoproteins involved in plateletadhesive responses. Am J Pathol 1986:124:324-34.

20. Woods VL Jr, Wolff LE, Keller DM. Resting platelets contain a substantial centrally located pool of glycoprotein IIb-IIla complex which may be accessible to some but not other extracellular proteins. J Biol Chem 1986;261:1.542-51

21. Ruoslahti E, Pierschbacher MD. Arg-Gly-Asp: a versatile cell recognition signal. Cell 1986;44:517-8,

22. Kloczewiak M, Timmons S, Hawiger J. Recognition site for the platelet receptor is present on the 15-residue carboxy-terminal fragment of the gamma chain of human fibrinogen and is not involved in the fibrin polymerization reaction. Thromb Res 1983;29:249-55.

23. Parise LV, Smyth SS, Coller BS. Platelet morphology, biochemistry, and function. In: Beutler $\mathrm{E}$, Lichtman MA, Coller B, Kipps TJ, Seligsohn U. (ed.) - Williams Hematology $6^{\text {th }}$ ed. The McGraw-Hill Companies, 2001. p. $1357-1408$

24. Ginsberg MH, Frelinger AL, Lam SC, Forsyth J, McMillan $R$, Plow EF, Shattil SJ. Analysis of platelet aggregation disorders based on flow cytometric analysis of membrane glycoprotein IIb-IIIa with conformation-specific monoclonal antibodies. Blood 1990;76:2017-23.
25. Warkentin TE, Powling MJ, Hardisty RM. Measurement of fibrinogen binding to platelets in whole blood by flow cytometry: a micromethod for the detection of platelet activation. Br J Haematol 1990;76:387-94.

26. Janes SL, Wilson DJ, Chronos N, Goodall AH. Evaluation of whole blood flow cytometric detection of platelet bound fibrinogen on normal subjects and patients with activated platelets. Thromb Haemost 1993;70:659-66.

27. Fijnheer R, Pietersz RN, de Korte D, Gouwerok CW, Dekker WJ, Reesink HW, Roos D. Platelet activation during preparation of platelet concentrates: comparison of the platelet-rich plasma and the buffy coat methods. Transfusion 1990;30:634-8.

28. Nurden P. Bidirectional trafficking of membrane glycoproteins following platelet activation in suspension. Thromb Haemost 1997;78:1.305-15.

29. Lozano M, Escolar G, Mazzara R, Connor J, White JG, DeLecea $C$, O rdinas A. Effects of the addition of secondmessenger effectors to platelet concentrates separated from whole-blood donations and stored at 4 degrees C or -80 degrees C. Transfusion 2000;40:527-34.

30. Escolar G, White JG. Changes in glycoprotein expression after platelet activation: differences between in vitro and in vivo studies. Thromb Haemost 2000;83:371-86.

31. Kroll MH, Hellums JD, McIntire LV, Schafer AI, Moake JL. Platelets and shear stress. Blood 1996:88:1.525-41.

32. Clemetson KJ, Clemetson JM. Platelet GPIb-V-IX complex Structure, function, physiology, and pathology. Semin Thromb Hemost 1995;21:130-6.

33. Modderman PW, Admiraal LG, Sonnenberg A, von dem Borne AE. Glycoproteins $V$ and $I b-I X$ form a noncovalent complex in the platelet membrane. J Bio Chem 1992;267:364-9.

34. Andrews RK, Shen Y, Gardiner EE, Dong JF, Lopez JA, Berndt MC. The glycoprotein Ib-IX-V complex in platelet adhesion and signaling. Thromb Haemost 1999;82:357-64.

35. Andrews RK, Munday AD, Mitchell CA, Berndt MC. Interaction of calmodulin with the cytoplasmic domain of the platelet membrane glycoprotein Ib-IX-V complex. Blood 2001;98:681-7.

36. Gu M, Xi X, Englund GD, Berndt MC, Du X. Analysis of the roles of 14-3-3 in the platel et glycoprotein Ib-IX mediated activation of integrin alpha(IIb)beta(3) using a reconstituted mammalian cell expression model. J Cell Biol 1999;147:1.085-96.

37. Pedrazzoli $P$, Noris $P$, Perotti C, Schiavo R, Ponchio L, Belletti $S$, et al. Transfusion of platelet concentrates cryopreseved with ThromboSol plus low-dose dimethylsulphoxide in patients with severe thrombocytopenia: pilot study. Br J Haematol 2000;108:653-59.

38. Lozano ML, Rivera J, Corral L, Gonzalez-Conejero R, Vicente V. Platelet cryopreservation using a reduced dimethyl sulfo xide concentration and second-messenger effectors as cryopreserving solution. Cryobiology 1999;39:1-12.

39. van Zanten GH, Heijnen HFG, Wu Y, Shut-Hese KM, Slootweg PJ, de Groot PG, et al. A fifty percent reduction 
of platelet surface glycoprotein lb does not affect platelet adhesion under flow conditions. Blood 1998; 91:2.353-59.

40. Hamburger SA, McEver RP. GMP-140 mediates adhesion of stimulated platelets to neutrophils. Blood 1990;75: 550-4.

41. Handa K, Nudelman ED, Stroud MR, Shiozawa T, Hakomori S. Selectin GMP-140 (CD62; PADGEM) binds to sialosyl-Le(a) and sialosyl-Le(x), and sulfated glycans modulate this binding. Biochem Biophys Res Commun 1991; 181:1.223-30.

42. Hsu-Lin $S$, Berman $C L$, Furie BC, August D, Furie B. A platelet membrane protein expressed during platelet activation and secretion. Studies using a monoclonal antibody specific for thrombin-activated platelets. J Biol Chem 1984;259:9121-6.

43. Johnston GI, Cook RG, McEver RP. Cloning of GMP-140, a granule membrane protein of platelets and endothelium: sequence similarity to proteins involved in cell adhesion and inflammation. Cell 1989;56:1.033-44.

44. McEver RP, Martin MN. A monoclonal antibody to a membrane glycoprotein binds only to activated platelets. J Biol Chem 1984;259:9.799-804.

45. Larsen E, Celi A, Gilbert GE, Furie BC, Erban JK, Bonfanti $R$, Wagner DD, Furie B. PADGEM protein: a receptor that mediates the interaction of activated platelets with neutrophils and monocytes. Cell 1989;59:305-12.

46. Michelson AD, Barnard MR, Hechtman HB, MacGregor $\mathrm{H}$, Connolly RJ, Loscalzo J, Valeri CR. In vivo tracking of platelets: circulating degranulated platelets rapidly lose surface P-selectin but continue to circulate and function. Proc Natl Acad Sci USA 1996;93:77-82.

47. Kostelijk EH, Fijnheer R, Nieuwenhuis HK, Gouwerok $\mathrm{CW}$, de Korte D. Soluble P-selectin as parameter for platelet activation during storage. Thromb Haemost 1996;76:1.086-9.

48. Nieuwenhuis HK, van Oosterhout JJ, Rozemuller $E$, van Iwaarden F, Sixma JJ. Studies with a monoclonal antibody against activated platelets: evidence that a secreted 53,000-molecular weight lysosome-like granule protein is exposed on the surface of activated platelets in the circulation. Blood 1987;70:838-45.

49. Miao WM, Vasile E, Lane WS, Lawler J. CD 36 associates with $C D 9$ and integrins on human blood platelets. Blood 2001;97:1.689-96.

50. Israels SJ, Gerrard JM, Jacques YV, McNicol A, Cham $B$, Nishibori M, Bainton DF. Platelet dense granule membranes contain both granulophysin and P-selectin (GMP-140). Blood 1992;80:143-52.

51. Lozano M, Escolar G, Mazzara R, Connor J, White JG, DeLecea C, Ordinas A. Effects of the addition of secondmessenger effectors to platelet concentrates separated from whole-blood donations and stored at 4 degrees C or -80 degrees C. Transfusion 2000;40:527-34.

52. Thibert V, Bellucci S, Edelman L, Tandon NN, Legrand C. Quantitation of platelet glycoprotein IV (CD36) in healthy subjects and in patients with essential thrombocythemia using an immunocapture assay. Thromb Haemost 1992;68:600-5.

53. Diaz-Ricart M, Tandon NN, Carretero M, Ordinas A Bastida $E$, Jamieson $G$ A. Platelets lacking functional CD36 (glycoprotein IV) show reduced adhesion to collagen in flowing whole blood. Blood 1993;82:491-6.

54. Febbraio M, Silverstein RL. Identification and characterization of LAMP-1 as an activation-dependen platelet surface glycoprotein. J Biol Chem 1990;265:31-7.

55. Michelson AD, Rajasekhar D, Bednarek FJ, Barnard $M R$. Platelet and platelet-derived microparticle surface factor $\mathrm{V} / \mathrm{Va}$ binding in whole blood: differences between neonates and adults. Thromb Haemost 2000; 84:689-94.

56. Zwaal RF, Schroit AJ. Pathophysiologic implications of membrane phospholipid asymmetry in blood cells. Blood 1997;89:1.121-32.

57. Mann KG, Nesheim ME, Church WR, Haley $P$, Krishnaswamy $\mathrm{S}$. Surface-dependent reactions of the vitamin K-dependent enzyme complexes. Blood 1990;76:1-16.

58. Matsubayashi H, Weidner J, Miraglia CC, Mclntyre JA. Platelet membrane early activation markers during prolonged storage. Thromb Res 1999;93:151-60.

59. Gao DY, Neff K, Xiao HY, Matsubayashi $H$, Cui XD, Bonderman $P$, Bonderman D, Harvey K, McIntyre JA Critser J, Miraglia CC, Reid T. Development of optimal techniques for cryopreservation of human platelets. I. Platelet activation during cold storage (at 22 and 8 degrees C) and cryopreservation. Cryobiology 1999; 8:225-35.

60. Abrams C, Shattil SJ. Immunological detection of activated platelets in clinical disorders. Thromb Haemost 1991;65:467-73.

61. Cramer EM, Lu H, Caen JP, Soria C, Berndt MC, Tenza D. Differential redistribution of platelet glycoprotein Ib and IIb-IIla after plasmin stimulation. Blood 1991; 77:694-9.

Recebido: 07/03/02

Aceito: $11 / 11 / 02$ 